\title{
Influence of the Utilized Voltage and Feed Rate on Physical Properties of 3D-Tubular Construction Nanofibers by Using Advers Charge Electrospinning Method
}

\author{
Salih Abbas Habeeb \\ Polymers and Petrochemical Industries Laboratory, Faculty of Materials Engineering, \\ University of Babylon, Hillah, Iraq
}

\begin{abstract}
This research explains the fabrication of polyacrylonitrile tubular construction nanofibers by using against the charge electrospun process and important of the constant working parameters such as high polymer concentration and high rotational speed mandrel collector with utilized voltage and feed rate to perform the effect of each one on the diameter, adjustment, porosity, average surface area and average pore size of nanofibers. Results showed that the growth in the flow rate of the polymer solution considerably increased the nanofibers diameter, a scale of the average diameters, the nanofibers diameter arrangement were regularly joining. Otherwise, the nanofibers diameter, the scale of the average diameters reduced and the nanofibers diameter arrangement have been regularly smaller as the applied voltage raised. While changing both the utilized voltage and feed rate of polymer solution leads to improve the porosity percentage, heights intensity $(\mathrm{Y} \%)$, average surface area $\left(\mathrm{nm}^{2}\right)$ and limited the average pore size $(\mathrm{nm})$.
\end{abstract}

Key words: Opposite charge electrospinning, tubular structure, mean surface area, flow rate, utilized voltage, heights intensity

\section{INTRODUCTION}

The generation rate of nanofibres from a single needle setup or traditional electrospinning method is considerably low and limiting the large-scale application comparied with multi-needles (Alghoraibi and Alomari, 2018), therefore, for production aligned fibers must be applied to the different kinds of collectors such as rotating drums or mandrel collectors (SalehHudin et al., 2018). Because the low generation rate and non-oriented nanofiber production plays a negative role in the development of the electrospinning method (Rafiei et al., 2013; Savva and Krasia-Christoforou, 2018). The rotating collector can be applied to manufacture the 3D nanofibers by using the rotation speed which supports a serious role in limiting the degree of anisotropy. Furthermore, the geometry of the collector can be chosen according to the application. For example, a cylindrical can be used to form a film while a rotating mandrel can be used to produce a tubular structure (Sill and von Recum, 2008; Yuan et al., 2018). One of the important advantages of using a mandrel or drum collector is a fast drying process occurrence that can prevent the fusion at the contact sites and keep the original fiber shape (Shim et al., 2010). Also, these fibers accumulated at a higher drum or mandrel rotation speed have a closer arrangement of fiber alignment with high porosity (Xiang et al., 2011; Jin et al., 2018; Savva and
Krasia-Christoforou, 2018). To fabricate fibers that are not only non-woven meshes but also aligned, patterned, twisted yarn and three-dimensional structures, developing new techniques for controlling the deposition behavior of the fibers either by mandrel collector shaft, rotating drums, disk collectors or parallel electrodes are required used the reverse charge nozzle's to align 3-D nanofibers into a uniaxial array (Dabirian and Hosseini, 2009; Xie et at., 2010; Chen et al., 2017) .

They modified the typical set-up by installing two needles in reverse directions and pumping the polymer solution into needles by two syringe liquid pumps with the same injection rate (Xie et al., 2010). The polyacrylonitrile nanofibers that are geometrically aligned and oriented are prepared using an unconventional method, using two needles in two opposite positions and an orthogonal rotational collector with the needle axis (Mottaghitalab and Haghi, 2011). The electrospinning parameters such as polymer concentration, applied voltage and solution flow rate strongly affects the rate of solidification in the resulting nanofibers. The rapid expansion and fastening of the polymer chains lead to another important effect: the low crystallization of the polymer nanofibers, since, the stretched chains do not have enough time to form the crystal. However, these non-crystalline polymer chains in nanofibers are strongly oriented (Zong et al., 2002; Garg and Bowlin, 2011). The 
electrical applied voltage is the most important factor of the electrospinning method which directly affects the dynamics of the fluid. In the electrospinning method, the polymer solution is equipped with high voltages to increase the electrical charge of the polymer solution. However, the changes in applied voltage, reflected in the droplet shape suspended in the spindle nozzle (Jacobs et al., 2010; Stocco et al, 2018). Taking into account the effects of electrospinning factors on the diameter of the nanofibers and keeping the other factors constant. The diameter of the nanofibers decreases with the increased electrical applied voltage on the polymer solution (Panda, 2007; Ren, 2013; Stocco et al, 2018). The expansion in the applied voltage (i.e., by increasing the electric field strength), raises the electrostatic opposing force on the fluid jet which ultimately chooses the reduction of fiber diameter (Deitzel et al., 2001; Ren, 2013; Rogina, 2014). Increases the applied voltage due to increasing the temporary forces that try to align the nanofibers in spite of increases whipping instability of electrospinning jet (Jalili et al, 2006). In addition, the increase the electric forces lead to enhance a surface area of the jet accelerated the evaporation rate of the solvent, decreasing the radius loop and slower elongation rate of the electrospinning jet at higher polymer concentrations (Thompson et al., 2007). On the other hand, the rising flow rate due to improving the production rate and diameter of the fibers (Dippold et al., 2017; Wang et al., 2017), also at higher flow rates as more materials are drawn from the nozzle tip and the time for the jet to dry is reduced (Garg and Bowlin, 2011). Fiber morphology is controlled by experimental design and depends on solution delivery, a polarity of the solvent, solution concentration, polymer viscosity, molecular weight an applied voltage (Jalili et al., 2006; Shalumon et al., 2018). Therefore, using the reverse charge electrospinning method of spinning in polyacrylonitrile/DMF solution. In order to produce three-dimensional nanomaterials with good morphological properties, electrical parameters such as applied voltages and polymer flow rate, rapid vapor evaporation of the solvent should controlled while the jet is accelerated towards the assembly drive to improve the electrospinning of the polymer solution (Shim et al., 2010). By Bonani et al. (2011) developed a new duplicate electrospinning system for producing the biohybrid nanofiber constructs they found high results in anisotropic properties of tubular structure nanofibers by using this system. Therefore, one of the aims in this research is to create a structure that reproduces anisotropic properties by collection of the collector shape and the collector rotation enables to produce a tubular structure with tunable anisotropic properties by using the highly concentrated polymer solutions in electrospinning. It offers a unique collection of control over tubular structure nanofibrous at approximately high production rates and increased the percentage of porosity, mean pores diameter with standard deviation and average surface area with standard deviation.

\section{MATERIALS AND METHODS}

Commercial Polyacrylonitrile (PAN) powder with a molecular weight of $100,000(\mathrm{~g} / \mathrm{mol})$ was supplied by the Poly Acryl Company, Iran and Dimethyl Formaldehyde (DMF) was obtained from Merck (Schuchardt OHG 85662 Hchenbrunn, Germany).

Product of the electrospinning polymeric solutions: The appropriate amounts of PAN and DMF mixed using a heating magnetic stirrer at a temperature of $40^{\circ} \mathrm{C}$ for $4 \mathrm{~h}$ to obtain a well-dissolved transparent light yellow homogeneous polymer solution, ready schematic diagram of the experimental setup shown (Fig. 1). To produce the corresponding to the electrospinning provisions used for production tubular structure nanofibrous by the influence of the flow rate of polymer solution and applied voltage are already in Table 1.

"Scanning Electron Microscopy" (SEM) and nanofibers morphology analysis: The structural and morphological characterizations of the pure polymeric structures were done by Scanning Electron Microscopy (SEM), KYKY-EM 3200 Model, the surface of specimens was spread with a layer of gold with the thickness of $100 \AA$ by using the sputter coater KYKY-SBC12, the mean diameter with Standard Deviation (SD) calculated by using Digimizer image analysis software Version 5.3.3 and the frequency histograms of the distribution of nanofibers diameter was plotted by the XLSTAT analysis Software, 2016 for Microsoft Excel versions.

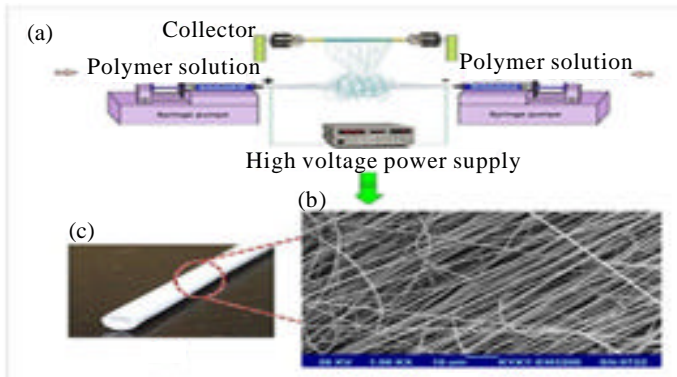

Fig. 1: a) Schematic diagram of the adverse charge electrospinning set-up fpr aligning nanofibers; $b$ ) $1.00 \mathrm{KX} 1,10 \mu \mathrm{m}$ SEM images and c) Tubular structure sample 
Table 1: The electrospinning conditions used for production tubular structure nanofibrous by the effect of the applied voltage and flow rate of polymer solution Utilized voltage electrospun conditions

Applied Voltage change (kV)

Polymer concentration (wt.\%)

Rotational speed (rpm)

Values

$12,16,20$

Feed rate electrospun conditions

Values

Applied Voltages (kV)

15.00

4328.0

Polymer concentration (wt.\%)

20.00

Rotational speed (rpm)

15.00

Distance between the needles and collector $(\mathrm{cm}) \quad 20.00$

Distance between two opposite needles $(\mathrm{cm}) \quad 10.00$

Flow rate of solution $(\mathrm{mL} / \mathrm{h})$

00.5

Distance between the needles and collector $(\mathrm{cm})$

4328.0

Distance between two opposite needles $(\mathrm{cm}) \quad 10.00$

Flow rate of solution change $(\mathrm{mL} / \mathrm{h})$

$0.3,0.4,0.5$

Statistical analysis: All the data were analyzed regarding to Kruskal-Wallis test (XLSTAT Analysis Software, 2016 for Microsoft Excel Versions) to determine if the data were normally or non-normally distributed. The data from which all of the specimens extracted follows a normal distribution. Data of pore diameter distribution according to statistical (one-way ANOVA) indication did allow at $\mathrm{p}<0.05$.

Alignment of nanofibrous analysis: "Fast Fourier Transform" (FFT) method was used to identify the functional set and vibrational modes of the materials as well as to quantify the alignment of nanofibers. SPIP (TM) Version 6.7.4 image metrology A/S, Horsholm, Denmark software analysis (Scanning probe image processor) converted to 8-bit grayscale TIF files and typical images are $256 \times 256$ or $512 \times 512$ pixels for the FFT/PSD analysis used to calculate the fast "Fourier transform" and power spectrum density with two-dimensional is calculated as the power value normalized with the area size of each element. PSD $(u, v)=P(u, v) /\left(x\right.$ Range ${ }^{*} y$ Range $)$. Where $x$ Range and $y$ Range are physical extents of the source image and $(\mathrm{u}, \mathrm{v})$ are $\mathrm{u}, \mathrm{v}$ the discrete Fourier indexes $=0$, 1, 2. For $1 \mathrm{D}$ average $Y-P S D$, The PSD Line medium uses will determine PSD spectra for each Y column and then determine the medium PSD spectrum. Here, each $Z$ value is calculated as the power value normalized with are the size of each element. PSD $(u)=P(u)$ range where $P(u)$ is the power value for the Fourier index you and range is the physical range of the profiles if the graph will possess a higher accuracy it must be calculated in high-resolution mode and the four highest peaks have been detected (M1-M4).

Surface areas and porosity of nano fibers analysis: There are some ways of testing the porosity such as image analysis (Savva and Krasia-Christoforou, 2018) by using SPIP (TM) Version 6.7.4 image metrology $\mathrm{A} / \mathrm{S}$, Horsholm, Denmark software analysis (Scanning Probe Image Processor) to detect the pores in the SEM image and calculated the porosity (\%), average surface area (NM) $2 \pm$ standard deviation, mean pores diameter $(\mathrm{NM}) \pm$ standard deviation and the heights intensity ( $\mathrm{Y} \%$ ) of distribution pores using the threshold method according to the following:
- Limited the inspection box on the surface of the image

- Detected the diameter of the average pore with Standard Deviation (SD) In addition, surface area with (SD) On the surface of image

- Plotted the histogram with limited the heights intensity of pore distribution (Y\%) and porosity $\%$

\section{RESULTS AND DISCUSSION}

Influence of the electrospinning parameters on nanofibers diameter: The applied electric field is one of the most essential parameters in the electrospinning method due to its direct influence on the dynamics of the fluid flow. The adverse charge set-up showed a set of experiments with the high-applied voltage varying from $12-16$ and to $20 \mathrm{kV}$. The other parameters were put fixed for the purpose of comparison were shown in the (Table 1). Figure 2 explains the medium fiber diameter with a Standard Deviation (SD) produced from 15 wt.\% Polymer solutions produced by the reverse charge set-up as a function of the three applied voltages. And Fig. 3 present the SEM micrographs and frequency histogram of the distribution of the nanofibers diameter at $15 \mathrm{wt} \%$ of PAN solution concentration for three different applied voltages. From the Fig. 2 it can be noted that on extending in the applied voltage. Due to decrease the average diameter of the nanofibers which can be associated with the expanded the effect of pulling force two needles. Other researchers observed this

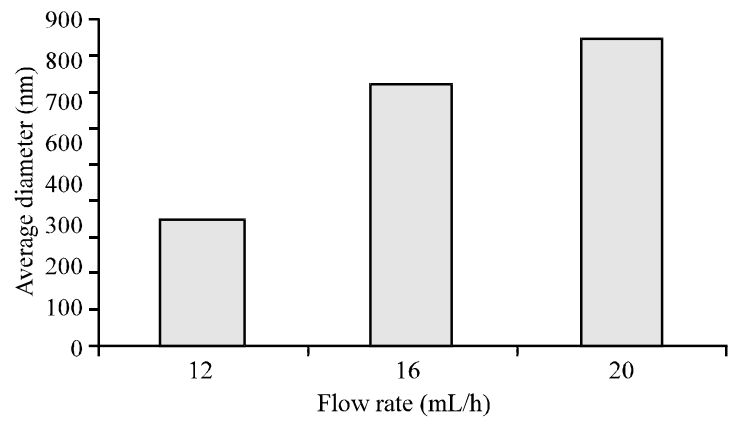

Fig. 2: The average fiber diameter produced from 15 wt.\%. Polymer solutions by the opposite charge set-up as a function of the three applied voltages 


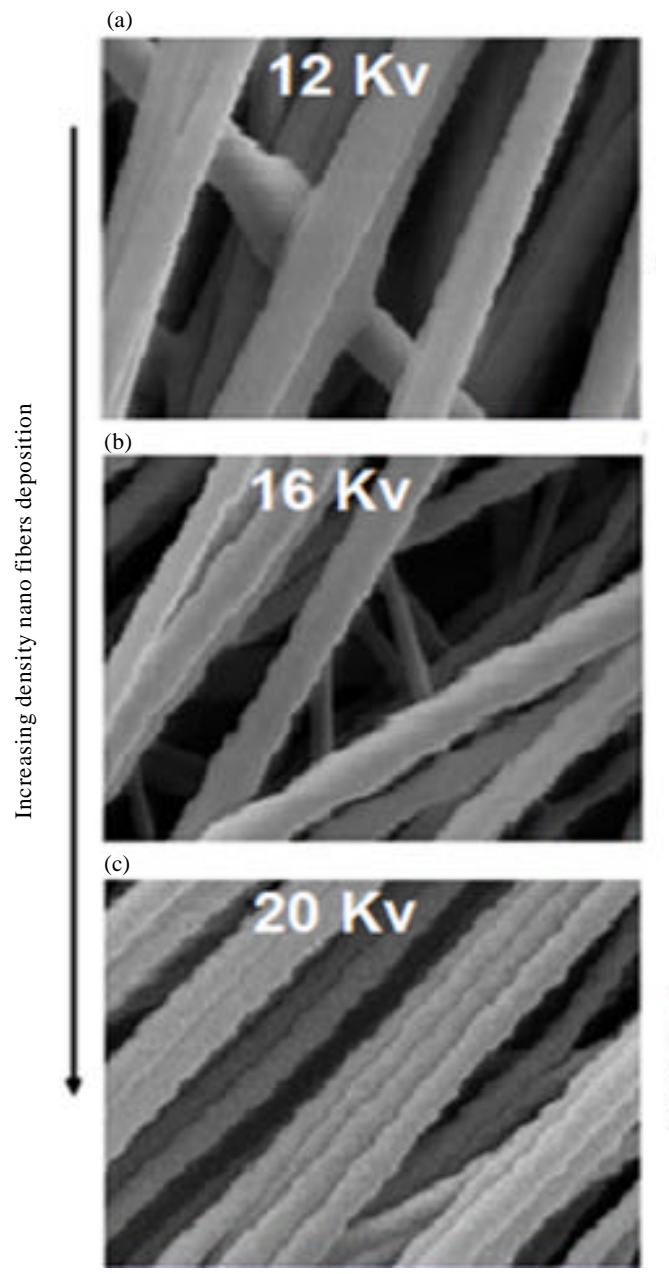

$10,00 \mathrm{KX}, 1 \mu \mathrm{m}$ SEM images
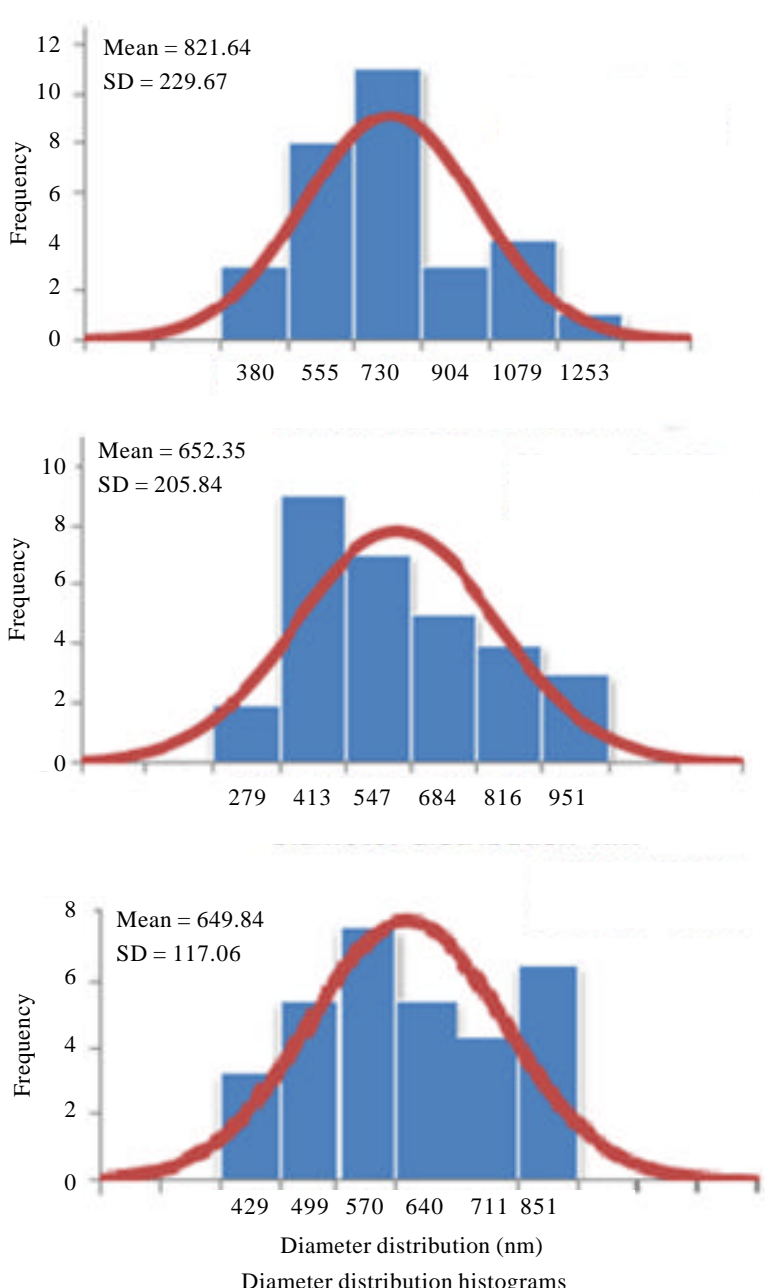

Fig. 3: a-c) SEM micrographs and the frequency histograms of nanofibers diameter distribution with the many polymer flow rates

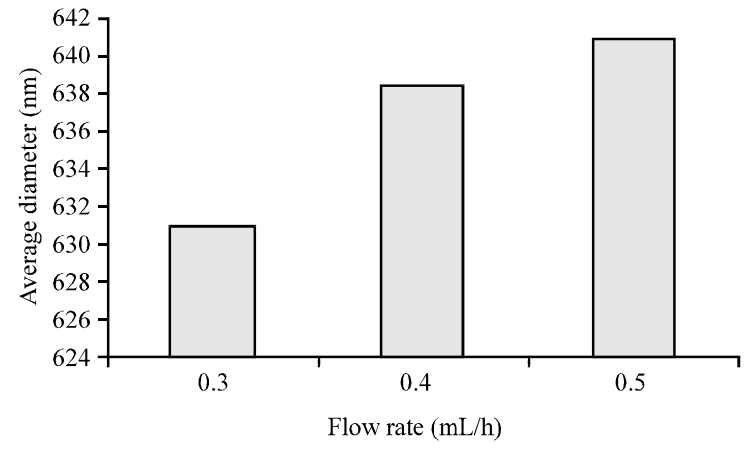

Fig. 4: Influence of the polymer feed on the nanofibers diameter at $4328 \mathrm{rpm}$ mandrel speeds

(Panda, 2007; Jacobs et al., 2010). Increasing the applied voltage due to quickening liquid jets from the tip of a capillary to collector and decreasing the time interval for manufacturing the nanofibers. Other researchers summarized this result as well (Deitzel et al., 2001; Ding et al., 2002; Boppa, 2009). Otherwise, from Fig. 3 it was noted that, rising in applied voltage of 12,16 and $20 \mathrm{kV}$ lead to variation in p-values until 0.113 , 0.04 and 0.027 which significate to the distribution of the nanofibers diameter locked to zero were deemed normal distribution and narrowing the nanofibers diameter distribution with uniform the solution concentrations and enhancing the production rate of aligned nanofibers (rising the density of nanofibers deposition) (Peterson, 2010) because the electrospinning jets produced by reverse charge system due to adding the mechanical force to electrical forces which provided a higher generation rate of nanofibers compared to single-jet electrospinning methods (Fig. 4). Therefore, the best voltage applied chosen as $20 \mathrm{kV}$ should be large 
quality fibers and high quantity of nanofibers precipitation. Considering the effect of the applied voltage on the medium diameter of PAN electrospun nanofibers manufactured at $15 \mathrm{wt} . \%$. Of polymer solution as the voltage raised, the fibers became smaller which compared with the previous studies performed by Lee et al. (2004) and Wu et al. (2010). The influence of the polymer flow rate on the nanofibers morphology was investigated by boosting it from 0.3 in $0.5 \mathrm{~mL} / \mathrm{h}$ in a set of experiments performed by using the inverse charge method where the following conditions were kept constant (Table 1). Figure 4 and 5 illustrate the influence of the polymer flow rate on the nanofibers diameter at the 4328-rpm mandrel speeds performed by inverse charge set-up and present the SEM micrographs with frequency histograms of the distribution of nanofibers diameter with the polymer flow rate of $0.3,0.4$ and $0.5 \mathrm{~mL} / \mathrm{h}$, respectively.

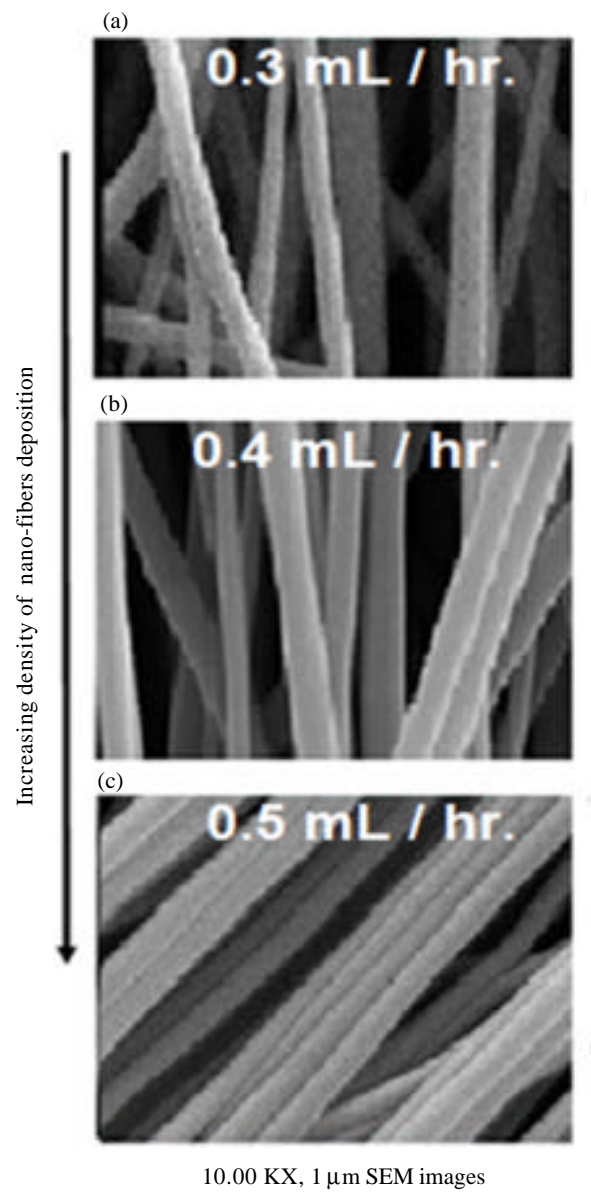

The obtained data as displayed in Fig. 4 and 5 well illustrate that, upon raising the solution flow rate, the electrospun nanofibers became thicker and more irregular. At higher flow rates, the hanging droplet at the end of the needle becomes larger and the solution jet can carry the fluid away with a faster velocity. From micrographs of SEM images (Fig. 5) The rise in the flow rate lead to raising the production rate of nanofibers (improve the frequency of precipitation nanofibers on rotating mandrel collector) which conforms with the summarized results from (Peterson, 2010). Some studies have regularly reviewed the relation between solution feed and fiber morphology. In usual it was determined that lowering flow rates produced fibers with thinner diameters (Zong et al., 2002; Wu et al., 2010).

The flow rate that was too high resulted in beading, since, fibers did not have an opportunity to dry prior to reaching the collector (Min et al., 2004; Wannatong et al.,
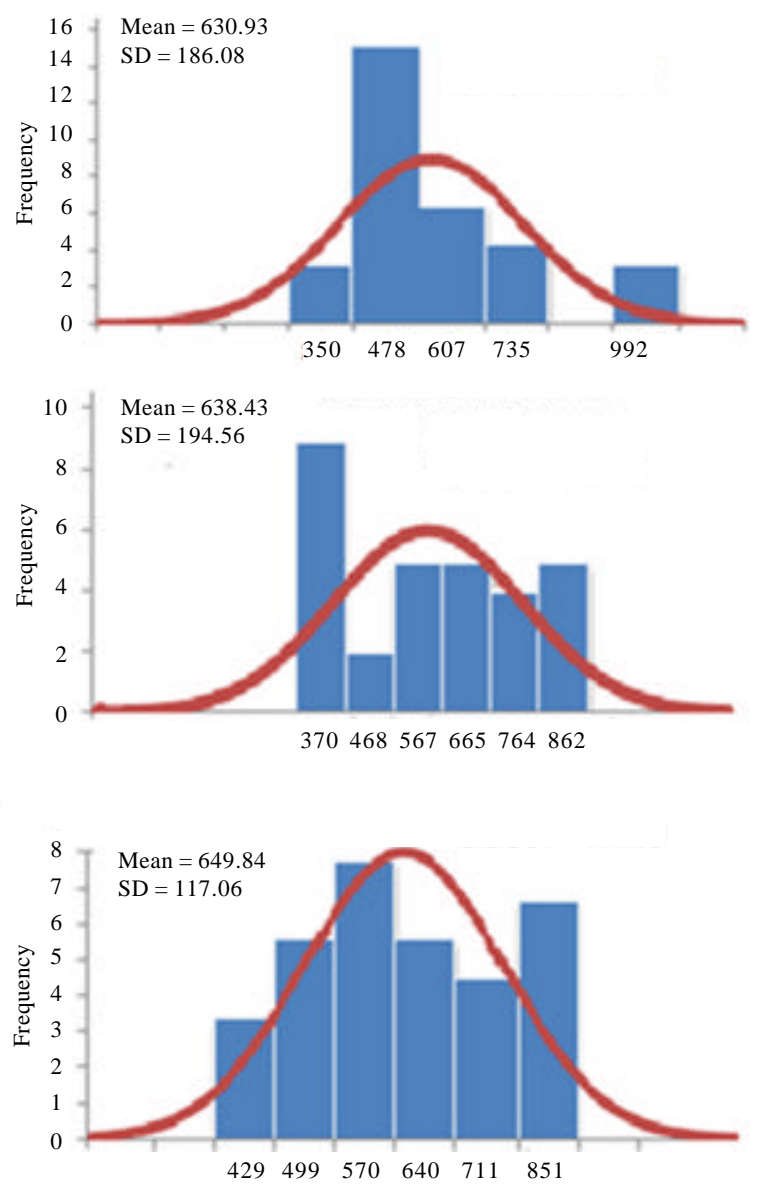

Diameter distribution histograms

Fig. 5: a-c) SEM micrographs and the frequemcy histograms of nanofibers diameter distribution with the many polymer flow rates 

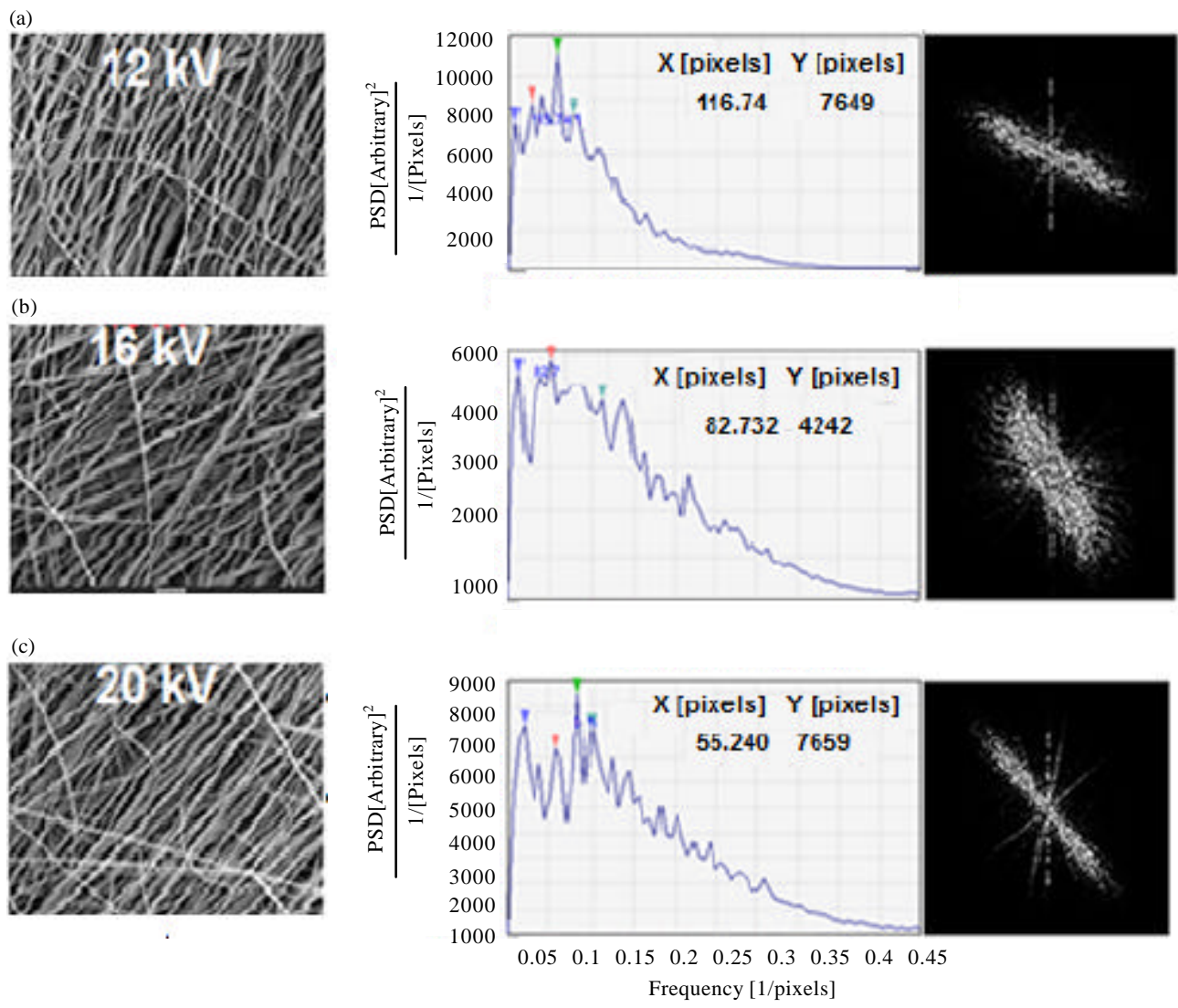

$1000,10 \mu \mathrm{m}$, SEM images

1- D PSD analysis

2D FFT/RSD analysis

Fig. 6: a-c) SEM micrographs, 1D average Y-PSD and 2D FFT/PSD analysis of SEM images of the nanofibers produced from the $15 \mathrm{wt} . \%$ polymer solutions at many applied voltages

2004; Zhang et al., 2005). The laboratory data acquired in this research explain that, the distribution of nanofibers diameter is progressively broader with increased flow rate of the solution from $0.3-0.5 \mathrm{~mL} / \mathrm{h}$ and the p-values change from $0.34,0.273$ and 0.027 according to Kruskal-Wallis testing method which corresponds with the summarized results from (Subbiah et al., 2005). The best result at $0.5 \mathrm{~mL} / \mathrm{h}$. Having the good quality of nanofibers and high frequency of nanofibers depositions.

Effect of the electrospinning parameters on alignment of nanofibers: A group of tests was performed by the opposite charge set-up with the high applied voltage varying from $12-16$ and to $20 \mathrm{kV}$. The other parameters kept constant for the purpose of comparison. Figure 6 shows that the SEM micrographs, the average heights intensity (Y)-Power Spectrum Density (PSD) and 2-dimensional Fast Fourier Transform (FFT) per power spectral density for analysis of SEM images (Lai et al., 2011; Kim et al., 2016) of the nanofibers produced from the $15 \mathrm{wt} \%$ polymer solutions in three different applied voltages obtained through opposite charge electrospinning method. In 1D Average Y-PSD testing (Fig. 6), the first and highest peak reflects the pitch distance which was changed from $115.74,82.732$ and 55.242 pixels and the average Power Spectrum Density (PSD) were changed from 7649, 4243 and 7659, respectively where the applied voltage varied from 12,16 and $20 \mathrm{kV}$. The FFT test of the SEM images of the nanofiber specimens used to convert the anisotropy of the scaffolds to digitize the alignment level of the nanofibers. Patterned, grayscale pixels distributed in the output image of the 2D FFT/PSD analysis to reflect the degree of fiber alignment of the original data image. The FFT data of the image with aligned fibers results in an output image with non-randomly and elliptically distribution pixels at 12,16 and $20 \mathrm{kV}$. The pixel intensities plotted between $0-360^{\circ}$ and the degree of alignment in the FFT data reflected the shape and height of the peak, also, the increased the intensity and fewer 

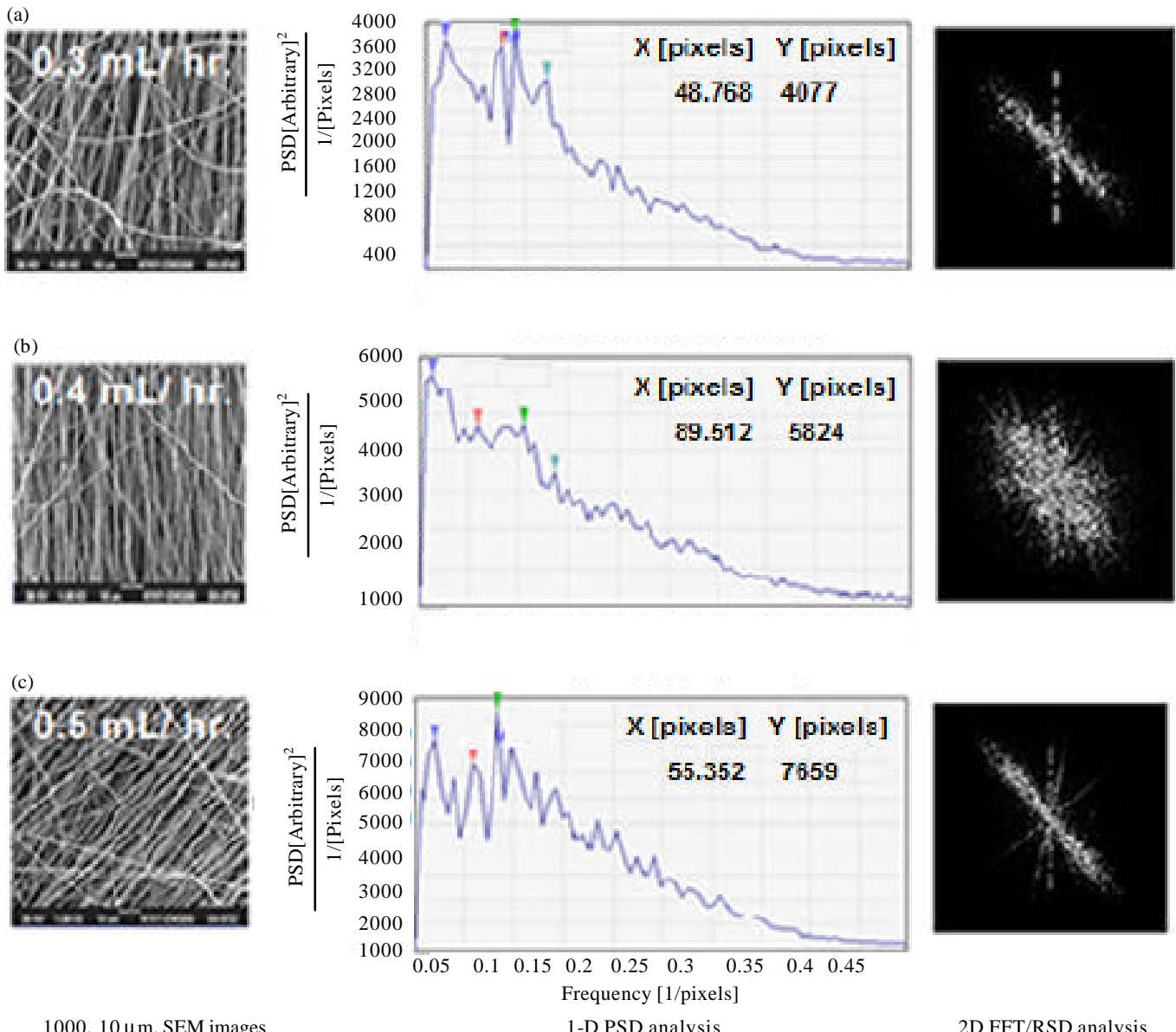

$1000,10 \mu \mathrm{m}$, SEM images

1-D PSD analysis

Fig. 7: a-c) SEM micrographs, 1D average Y-PSD and 2D FFT/PSD fourier images analysis of SEM images of the nanofibers produced from the $15 \mathrm{wt} . \%$ polymer solution at $4328 \mathrm{rpm}$ and at many polymer flow rates

occurrences of peaks indicate that the slightly alternating alignment with voltage because the directional electric field intensity toward the electrodes lead to align nanofibers and increase the bending instability the optimum results of the nanofibers alignment are presented in the $20 \mathrm{kV}$. The influence of voltage spotted in these tests may be as regards to the "whipping" instability. The many leading elements operating during electrospinning is the fast increase of a whipping instability that creates bending and lets the electrical forces to stretch the jet (Rahmani et al., 2014). Raising the utilized voltage, rise the whipping instability and then raises the as-spun nanofibers texture which makes uniformity of nanofibers more complicated but moreover, raises the influential forces which try to uniform the nanofibers at $12 \mathrm{kV}$ and above. The current results obtained agree with the previous experiments reported by Jalili et al. (2006) and Rahmani et al. (2014). On the other hand, the effect of the polymer flow rate on the resulting nanofibers morphology was studied by increasing it from $0.3-0.5 \mathrm{~mL} / \mathrm{h}$ in a set of experiments performed by using the opposite charge method. Figure 7 shows that the SEM micrographs, 1D average Y-PSD and 2D FFT/PSD analysis of SEM images of the nanofibers produced from the $15 \mathrm{wt} \%$ polymer solution at $(0.3,0.4,0.5) \mathrm{mL} / \mathrm{h}$. Flow rates of polymer fabricated through the opposite electrospinning set-up.

According to Fig. 7, we noted that formation fours highest peaks with high resolution mode were varied the flow rate, the first and highest peak reflects the pitch distance which were changed from $48.768,89.512$ and 55.352 pixels and the average Power Spectrum Density (PSD) were changed from 4077, 5824 and 7659 when increased the flow rate from $0.3,0.4$ and $0.5 \mathrm{~mL} / \mathrm{h}$, respectively. Using the FFT test of the SEM images of the nanofiber specimens to describe the anisotropy of the nanofibers and digitize the uniformity level of the nanofibers. Patterned, grayscale pixels distributed in the 

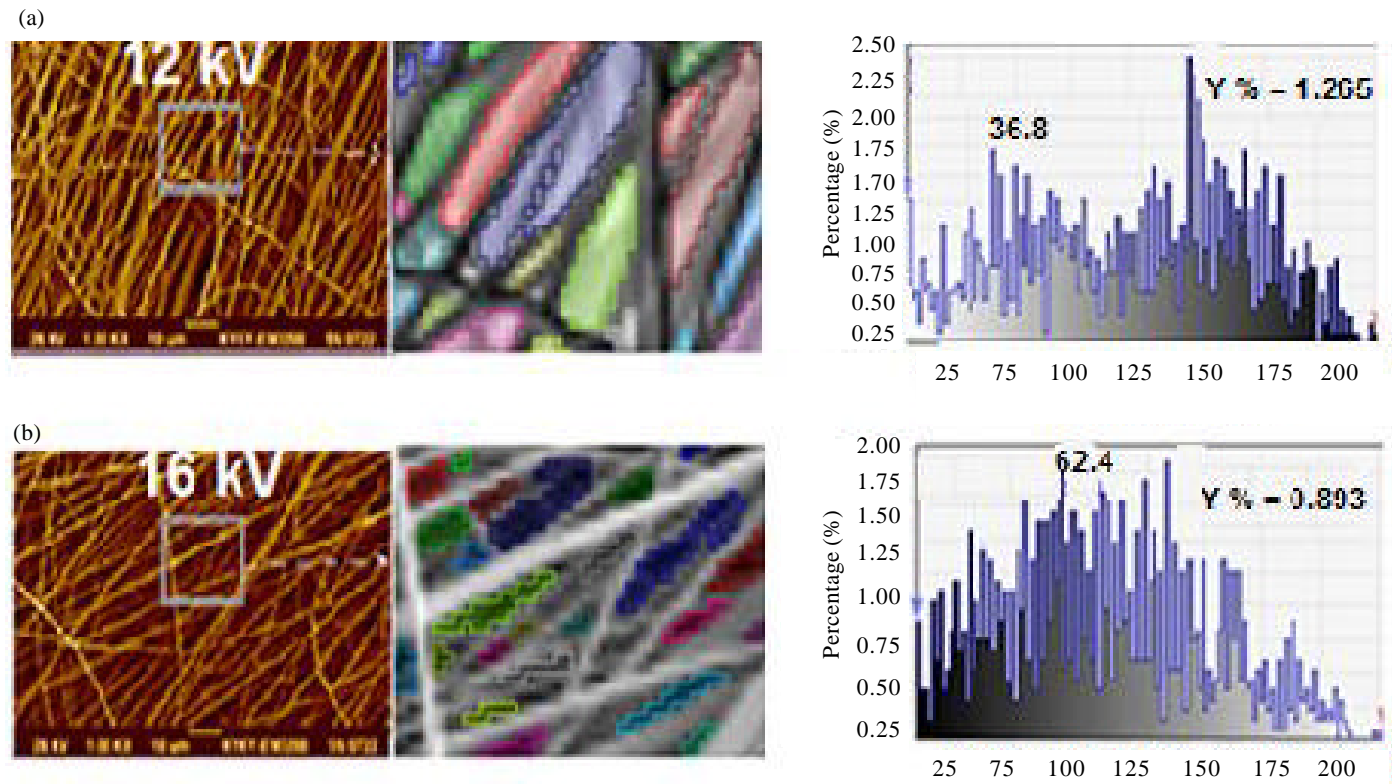

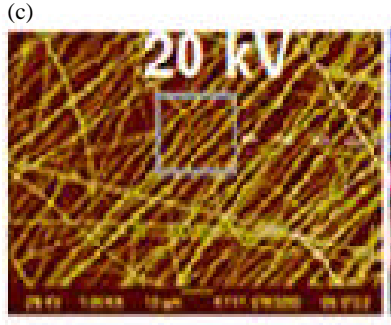

Image after thresholding

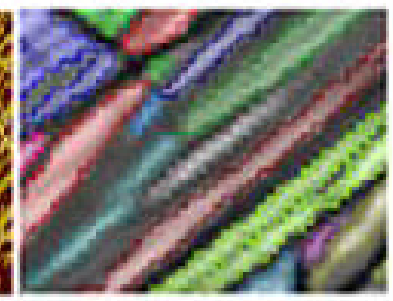

Detect pores in the box

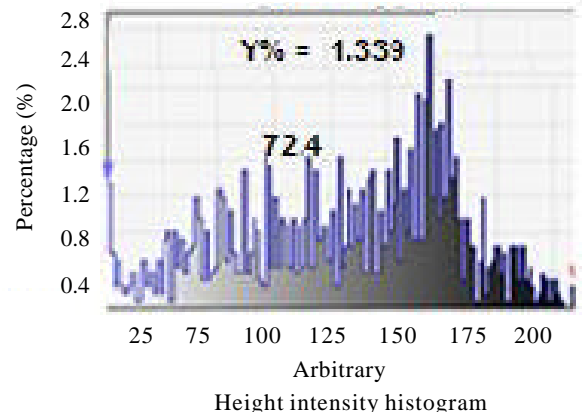

Fig. 8: SEM micrographs after thersholding, detected pores and height intensity histrogram analysis of the nanofibers produced from the $15 \mathrm{wt} . \%$ polymer solution at $4328 \mathrm{rpm}$ with many applied voltages, $\mathrm{N}=6, \mathrm{p}<0.05$

Table 2: The results of porosity (\%), mean surface area, mean pores diameter and height intensity of pores distribution (Y\%) by SPIP image processing software after effect of increased the applied voltage and flow rate of $15 \mathrm{wt} . \%$ of polymer concentration

\begin{tabular}{lcccc}
\hline Samples & Porosity $(\%)$ & Height intensity $(\mathrm{Y} \%)$ & Mean surface area $(\mathrm{nm})^{2}$ & Mean pore diameter $(\mathrm{nm})$ \\
\hline $12 \mathrm{kV}$ & 48.06 & 1.265 & $21940.2 \pm 81.45$ & $5.11 \pm 3.23$ \\
$16 \mathrm{kV}$ & 40.31 & 0.893 & $5371.9 \pm 71.16$ & $6.15 \pm 3.31$ \\
$20 \mathrm{kV}$ & 51.50 & 1.339 & $76983.1 \pm 98.16$ & $3.72 \pm 1.52$ \\
$0.3 \mathrm{~mL} / \mathrm{h}$ & 41.50 & 0.818 & $7618.5 \pm 8017.5$ & $7.00 \pm 3.41$ \\
$0.4 \mathrm{~mL} / \mathrm{h}$ & 44.10 & 0.372 & $6257.3 \pm 4541.3$ & $5.33 \pm 2.76$ \\
$0.5 \mathrm{~mL} / \mathrm{h}$ & 51.50 & 1.339 & $76983.1 \pm 98.16$ & $3.72 \pm 1.52$ \\
\hline
\end{tabular}

output image of the $2 \mathrm{D}$ FFT/PSD analysis to reflect the degree of fiber alignment of the original data image.

Effect of the electrospinning parameters on porosity and surface area of nanofibers: Figure 8 and 9 and Table 2 , show the effect of electrospinning parameters such as applied voltage and flow rate of polymer solution on porosity $(\%)$, mean surface area $(\mathrm{nm})^{2}$ with Standard Deviation (SD), mean pore diameter with Standard Deviation (SD) (nm), height intensity $(\mathrm{Y} \%)$ of pores distribution by using the threshold method to detect the pores in the SEM images with SPIP (TM) Software analysis (Catena et al., 2016; Tahalyani et al., 2018). When increased the applied voltage from 12,16 and $20 \mathrm{kV}$ slightly increased the percentage height, intensity of pore distribution, percentage porosity and the mean surface area while decreased the mean pore diameter with increased the applied voltage (Fig. 8, Table 2). The highest porosity $(\%)$, surface area $\left(\mathrm{nm}^{2}\right)$ and highest intensity ( $\mathrm{Y} \%$ ) of distribution pores on histogram curves and lowest mean pore size at $20 \mathrm{kV}$. The analyzed using 

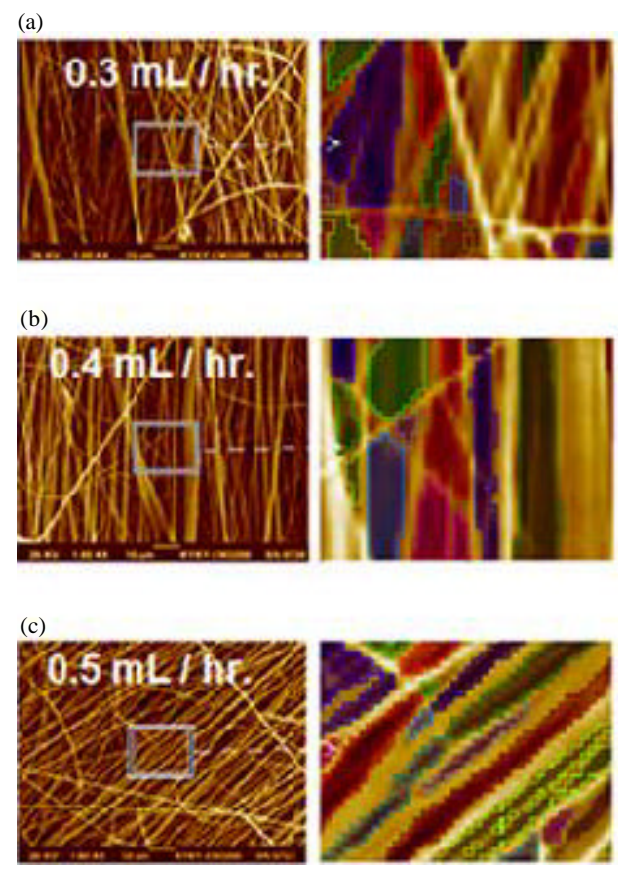

Images after thersholding

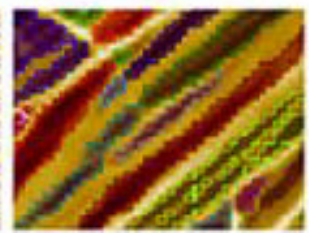

Detect pores in the box
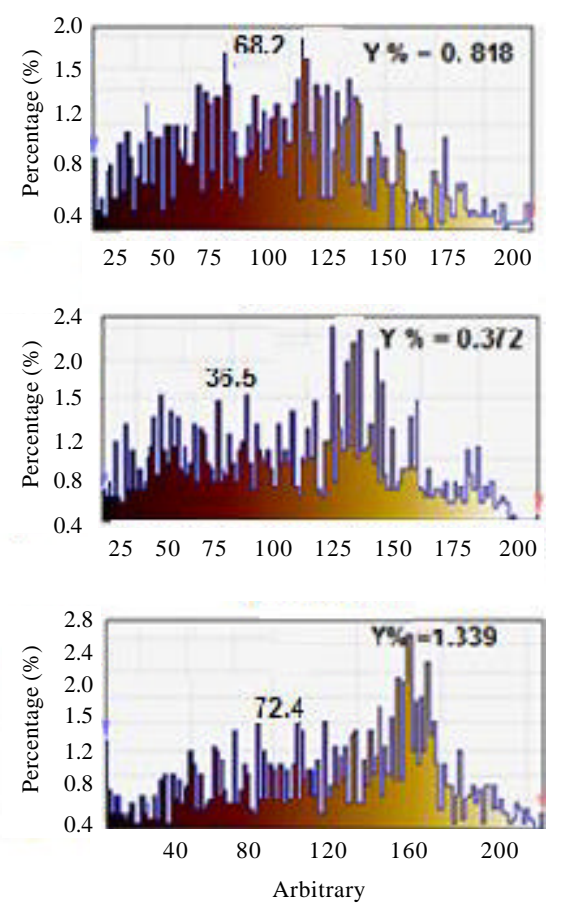

Height intensity histogram

Fig. 9: a-c) SEM micrographs after thersholding, detected pores and height intensity histogram analysis of the nanofibers produced from the $15 \mathrm{wt} . \%$ polymer solution at $4328 \mathrm{rpm}$ with many flow rates, $N=6, \mathrm{p}<0.01$

Kruskal-Wallis test (XLSTAT Analysis Software, one-way ANOVA) for the change in the pore size was not normally distributed. The statistical data reported about the changes in mean pore $\operatorname{size} \pm(\mathrm{SD})$ was accepted at $\mathrm{p}<0.05$ with increasing in the applied voltage.

On the others hand when increased the flow rate of polymer solution lead to increasing the porosity (\%), the mean surface area $(\mathrm{nm})^{2}$ and height intensity $(\mathrm{Y} \%)$ of distribution pores on histogram curves with decreased the mean pores diameter (nm) (Fig. 9, Table 2) and the changes in mean pore size $\pm(\mathrm{SD})$ with increased the flow rate was accepted at $\mathrm{p}<0.01$, the highest porosity (\%), surface area $(\mathrm{nm})^{2}$ and highest intensity (Y\%) of distribution pores on histogram curves at $0.5 \mathrm{~mL} / \mathrm{h}$. Therefore, we noted that, a polymeric nanofibers web containing the heights, surface area with a lowest pore size and pores diameter distribution become more narrowing with increasing the applied voltage and flow rate of polymer solution (Subbiah et al., 2005), also, at high polymer concentration and high rotational speed of mandrel collector we found a good relationship between the uniformity, aligned with porosity and surface area of nanofibers. Because controlling of the nanofibers deposition with rotating collector lead to linear straight jet by increased the flow rate especially at high concentration compared with the conventional electrospinning method (Zhou et al., 2015).

\section{CONCLUSION}

PAN tubular structures produced from highly aligned continuous and uniform nanofibers through obverse charge electrospinning methods. Raising the feed rate of the polymer solution considerably increased the average nanofibers diameter with Standard Deviation (SD) and the range of diameter of the nanofibers. The highest alignment, porosity (percentage), mean surface area $(\mathrm{nm})^{2}$, height intensity (Y\%) of pores distribution of nanofibers and lowest mean pores diameter $(\mathrm{nm})$ at $0.5 \mathrm{~mL} / \mathrm{h}$. Of polymer solution, among the three flow rates of polymer solutions studied. On the other hand, the diameter with Standard Deviation (SD) and range of nanofibers reduced as the utilized voltage increased and the highest alignment, porosity (\%) mean surface area $(n m)^{2}$, height intensity $(\mathrm{Y} \%)$ of pore distribution of nanofibers and lowest mean pore diameter (nm) at $20 \mathrm{kV}$ applied voltage, among the three applied voltages. The nanofibers produced from higher concentrations of polymer solutions as well as higher take-up speeds at 
higher flow rate and applied voltage as they had a higher directional alignment, mean surface area and porosity and lowest mean pore size.

\section{ACKNOWLEDGEMENTS}

The researchers are grateful to the Kefa Company at the Amirkabir University of Technology, Central Lab. University of Isfahan, Central Lab. at Kurdistan University, also, Polymers and Petrochemical Industries Labaratory at Babylon University.

\section{REFRENCES}

Alghoraibi, I. and S. Alomari, 2018. Different Methods for Nanofiber Design and Fabrication. In: Handbook of Nanofibers, Barhoum, A., M. Bechelany and A. Makhlouf (Eds.). Springer, Cham, Switzerland, ISBN:978-3-319-42789-8, pp: 1-46.

Bonani, W., D. Maniglio, A. Motta, W. Tan and C. Migliaresi, 2011. Biohybrid nanofiber constructs with anisotropic biomechanical properties. J. Biomed. Mater. Res. Part B. Appl. Biomater., 96: 276-286.

Boppa, V., 2009. Characterization of structure and tensile properties of Electrospun Web. MSc Thesis, NCSU Libraries, North Carolina, USA.

Catena, A., Q. Guo, M.R. Kunze, S. Agnello and F.M. Gelardi et al., 2016. Morphological and chemical evolution of gradually deposited diamond-like carbon films on polyethylene terephthalate: From subplantation processes to structural reorganization by intrinsic stress release phenomena. ACS. Appl. Mater. Interfaces, 8: 10636-10646.

Chen, J.Y., Q.J. Niu, G.K. Chen, J. Nie and G.P. Ma, 2017. Electrooxidation of methanol on Pt@ Ni bimetallic catalyst supported on porous carbon nanofibers. J. Phys. Chem. C., 121: 1463-1471.

Dabirian, F. and S.A. Hosseini, 2009. Novel method for nanofibre yarn production using two differently charged nozzles. Fibres Text East. Eur., 17: 45-47.

Deitzel, J.M., J. Kleinmeyer, D. Harris and N.C.B. Tan, 2001. The effect of processing variables on the morphology of electrospun nanofibers and textiles. Polymer, 42: 261-272.

Ding, B., H.Y. Kim, S.C. Lee, C.L. Shao and D.R. Lee et al., 2002. Preparation and characterization of a nanoscale poly (vinyl alcohol) fiber aggregate produced by an electrospinning method. J. Sci. Part B. Polym. Phys., 40: 1261-1268.

Dippold, D., A. Cai, M. Hardt, A.R. Boccaccini and R. Horch et al., 2017. Novel approach towards aligned PCL-Collagen nanofibrous constructs from a benign solvent system. Mater. Sci. Eng. C., 72: 278-283.
Garg, K. and G.L. Bowlin, 2011. Electrospinning jets and nanofibrous structures. Biomicrofluidics, 5: 013403-1-013403-19.

Jacobs, V., R.D. Anandjiwala and M. Maaza, 2010. The influence of electrospinning parameters on the structural morphology and diameter of electrospun nanofibers. J. Appl. Polym. Sci., 115: 3130-3136.

Jalili, R., M. Morshed and S.A.H. Ravandi, 2006. Fundamental parameters affecting electrospinning of PAN nanofibers as uniaxially aligned fibers. J. Appl. Polym. Sci., 101: 4350-4357.

Jin, L., B. Hu, S. Kuddannaya, Y. Zhang and C. Li et al., 2018. A three-dimensional carbon nanotube-nanofiber composite foam for selective adsorption of oils and organic liquids. Polym. Compos., 39: E271-E277.

Kim, J.I., T.I. Hwang, L.E. Aguilar, C.H. Park and C.S. Kim, 2016. A controlled design of aligned and random nanofibers for 3D bi-functionalized nerve conduits fabricated via a novel electrospinning set-up. Sci. Rep., 6: 1-12.

Lai, E.S., C.M. Anderson and G.G. Fuller, 2011. Designing a tubular matrix of oriented collagen fibrils for tissue engineering. Acta Biomater., 7: 2448-2456.

Lee, J.S., K.H. Choi, H.D. Ghim, S.S. Kim and D.H. Chun et al., 2004. Role of molecular weight of atactic poly (vinyl alcohol)(PVA) in the structure and properties of PVA nanofabric prepared by electrospinning. J. Appl. Polym. Sci., 93: 1638-1646.

Min, B.M., G. Lee, S.H. Kim, Y.S. Nam and T.S. Lee et al., 2004. Electrospinning of silk fibroin nanofibers and its effect on the adhesion and spreading of normal human keratinocytes and fibroblasts in vitro. Biomater., 25: 1289-1297.

Mottaghitalab, V. and A.K. Haghi, 2011. A study on electrospinning of polyacrylonitrile nanofibers. Korean J. Chem. Eng., 28: 114-118.

Panda, P.K., 2007. Ceramic nanofibers by electrospinning technique-A review. Trans. Indian Ceram. Soc., 66: 65-76.

Peterson, C.T., 2010. Hybrid nanomanufacturing process for high-rate polymer nanofiber production. MSc Thesis, University of Nebraska-Lincoln, Lincoln, Nebraska.

Rafiei, S., S. Maghsoodloo, B. Noroozi, V. Mottaghitalab and A.K. Haghi, 2013. Mathematical modeling in electrospinning process of nanofibers: A detailed review. Cellul. Chem. Technol., 47: 323-338.

Rahmani, S., M. Rafizadeh and F.A. Taromi, 2014. Statistical analysis of nanofibers alignment in magnetic-field-assisted electrospinning including an alignment percentage formula. J. Appl. Polym. Sci., Vol. 131, 10.1002/app.41179. 
Ren, C., 2013. PAN nanofibers and nanofiber reinforced composites. MSc Thesis, University of Nebraska-Lincoln, Lincoln, Nebraska.

Rogina, A., 2014. Electrospinning process: Versatile preparation method for biodegradable and natural polymers and biocomposite systems applied in tissue engineering and drug delivery. Appl. Surf. Sci., 296: 221-230.

SalehHudin, H.S., E.N. Mohamad, W.N.L. Mahadi and A.M. Afifi, 2018. Multiple-jet electrospinning methods for nanofiber processing: A review. Mater. Manuf. Processes, 33: 479-498.

Savva, I. and T. Krasia-Christoforou, 2018. Encroachment of Traditional Electrospinning. In: Electrospinning: From Basic Research to Commercialization, Kny, E., K. Ghosal and S. Thomas (Eds.). Royal Society of Chemistry, UK., ISBN:978-1-78801-100-6, pp: 24-54.

Shalumon, K.T., J. Anjana, U. Mony, R. Jayakumar and J.P. Chen, 2018. Process study, development and degradation behavior of different size scale electrospun poly (caprolactone) and poly (lactic acid) fibers. J. Polym. Res., Vol. 25,

Shim, I.K., M.R. Jung, K.H. Kim, Y.J. Seol and Y.J. Park et al., 2010. Novel three-dimensional scaffolds of poly (L-lactic acid) microfibers using electrospinning and mechanical expansion: Fabrication and bone regeneration. J. Biomed. Mater. Res. Part B. Appl. Biomater., 95: 150-160.

Sill, T.J. and H.A. von Recum, 2008. Electrospinning: Applications in drug delivery and tissue engineering. Biomater., 29: 1989-2006.

Stocco, T.D., N.J. Bassous, S. Zhao, A.E. Granato and T.J. Webster et al., 2018. Nanofibrous scaffolds for biomedical applications. Nanoscale, 10: 12228-12255.

Subbiah, T., G.S. Bhat, R.W. Tock, S. Parameswaran and S.S. Ramkumar, 2005. Electrospinning of nanofibers. J. Appl. Polym. Sci., 96: 557-569.

Tahalyani, J., S. Datar and K. Balasubramanian, 2018. Investigation of dielectric properties of free standing electrospun nonwoven mat. J. Appl. Polym. Sci., Vol. 135, 10.1002/app.46121.
Thompson, C.J., G.G. Chase, A.L. Yarin and D.H. Reneker, 2007. Effects of parameters on nanofiber diameter determined from electrospinning model. Polymer, 48: 6913-6922.

Wang, L., M.W. Chang, Z. Ahmad, H. Zheng and J.S. Li, 2017. Mass and controlled fabrication of aligned PVP fibers for matrix type antibiotic drug delivery systems. Chem. Eng. J., 307: 661-669.

Wannatong, L., A. Sirivat and P. Supaphol, 2004. Effects of solvents on electrospun polymeric fibers: Preliminary study on polystyrene. Polym. Intl., 53: 1851-1859.

Wu, H., J. Fan, C.C. Chu and J. Wu, 2010. Electrospinning of small diameter 3-D nanofibrous tubular scaffolds with controllable nanofiber orientations for vascular grafts. J. Mater. Sci. Mater. Med., 21: 3207-3215.

Xiang, P., M. Li, C.Y. Zhang, D.L. Chen and Z.H. Zhou, 2011. Cytocompatibility of electrospun nano?ber tubular scaffolds for small diameter tissue engineering blood vessels. Intl. J. Boil. Macromol., 49: 281-288.

Xie, J., M.R. MacEwan, A.G. Schwartz and Y. Xia, 2010. Electrospun nanofibers for neural tissue engineering. Nanoscale, 2: 35-44.

Yuan, T.T., A.M.D. Foushee, M.C. Johnson, A.R. Jockheck-Clark and J.M. Stahl, 2018. Development of electrospun chitosan-polyethylene oxide/fibrinogen biocomposite for potential wound healing applications. Nanoscale Res. Lett., 13: 1-12.

Zhang C., X. Yuan, L. Wu, Y. Han and J. Sheng, 2005. Study on morphology of electrospun poly (Vinyl Alcohol) mats. Eur. Polymer J., 41: 423-432.

Zhou, F.L., G.J. Parker, S.J. Eichhorn and P.L.H. Cristinacce, 2015. Production and cross-sectional characterization of aligned co-electrospun hollow microfibrous bulk assemblies. Mater. Charact., 109: 25-35.

Zong, X., K. Kim, D. Fang, S. Ran, B.S. Hsiao and B. Chu, 2002. Structure and process relationship of electrospun bioabsorbable nanofiber membranes. Polym., 43: 4403-4412. 